\title{
Outcomes of XEN Gel Stent Implantation in the Inferonasal Quadrant after Failed Trabeculectomy
}

\author{
Eyüp Düzgün ${ }^{1}$, Ali Olgun², Murat Karapapak ${ }^{3}$, Abdurrahman Alpaslan Alkan ${ }^{4}$, Melih Ustaoğlu $^{5}$
}

\begin{abstract}
Aim and objective: To evaluate the efficacy and safety of XEN stent implantation in the inferonasal quadrant after prior failed trabeculectomy. Materials and methods: Fourteen open-angle glaucoma patients with prior failed trabeculectomy were recruited to this retrospective study. Implantation of the stent was performed as a stand-alone procedure. The mean follow-up duration was 14.2 months. Best-corrected visual acuity, intraocular pressure (IOP), number of medications, complications, and the requirement for additional procedures were among the outcome measures recorded.

Results: Mean IOP reduced by $49.3 \%$ from $24.14 \pm 2.74 \mathrm{~mm} \mathrm{Hg}$ preoperatively to $12.23 \pm 2.89 \mathrm{~mm} \mathrm{Hg}$ at month $12(p<0.001)$. Medication usage reduced from $3.71 \pm 0.47$ medications preoperatively to $1.31 \pm 1.55$ at month $12(p=0.003)$. Adverse events included transient slight intracameral hemorrhage ( 5 eyes, $35.7 \%$ ), second trabeculectomy required ( 2 eyes, $14.3 \%$ ), and numerical hypotony (IOP $<5 \mathrm{~mm} \mathrm{Hg}$, in 3 cases, $21.4 \%)$, all of which resolved spontaneously. Six eyes (42.8\%) required postoperative bleb needling to further reduce IOP. There were no cases of vision loss, stent exposure, hypotony, lower eyelid malposition, bleb dysesthesia, or bleb-related infection.

Conclusion: XEN gel stent implantation in the inferonasal quadrant can be considered a viable surgical option for patients with a history of previously failed trabeculectomy requiring further IOP lowering.

Clinical significance: To the best of our knowledge, this is the first case series describing the outcome of inferonasal implantation of XEN gel stent following failed trabeculectomy.

Keywords: Inferonasal quadrant, Minimally invasive glaucoma surgery, Refractory glaucoma, Trabeculectomy, XEN gel stent.

Journal of Current Glaucoma Practice (2021): 10.5005/jp-journals-10078-1304
\end{abstract}

\section{INTRODUCTION}

The main objective of glaucoma surgery is to preserve visual function by preventing disease progression. In addition to traditional methods, several minimally invasive glaucoma surgery (MIGS) procedures have been described as a result of the quest for an ideal surgical procedure for glaucoma. Minimally invasive glaucoma surgery procedures have a good safety profile in mildto-moderate glaucoma but are less likely to achieve low intraocular pressure (IOP) needed for patients with advanced or refractory glaucoma. In addition, MIGS procedures had been anticipated to be less effective than traditional glaucoma filtering procedures and to be ineffective after conventional incisional surgery. ${ }^{1}$ The recently popular XEN gel implant (Allergan, Dublin, CA, USA) is one of the MIGS devices which creates an external drainage fistula in a similar concept to trabeculectomy without conjunctival or scleral dissection. The goal of the XEN surgery is to lower IOP through subconjunctival filtration of aqueous humor while minimizing the risk of complications associated with conventional glaucoma surgery. ${ }^{2}$

The XEN implantation has shown good results, including IOP control with the potential to keep the patient free of medication and the risk of complications was comparable to that of trabeculectomy. ${ }^{3,4}$ Therefore, it is claimed that the XEN gel stent can be used in patients with advanced and refractory glaucoma. ${ }^{1,4-7}$ Previous studies have reported successful results with XEN implantation in the superonasal quadrant in patients who had undergone failed prior glaucoma interventions. ${ }^{7-10} \mathrm{~A}$ recent comparative report has also shown that inferior implantation of XEN stent was as efficient as in the superior quadrant as a first-line intervention in naive glaucoma patients. ${ }^{11}$ However, no previous \begin{tabular}{l}
\hline \hline${ }^{1,3}$ Department of Ophthalmology, Sisli Hamidiye Etfal Training and \\
Research Hospital, University of Health Sciences, Istanbul, Turkey \\
${ }^{2}$ Department of Ophthalmology, Worldeye Hospital, Istanbul, Turkey \\
${ }^{4}$ Department of Ophthalmology, Ercis Sehit Rıdvan Cevik State \\
Hospital, Van, Turkey \\
${ }^{5}$ Department of Ophthalmology, Bursa Higher Specialization Training \\
and Research Hospital, University of Health Sciences, Bursa, Turkey \\
Corresponding Author: Abdurrahman Alpaslan Alkan, Department of \\
Ophthalmology, Ercis Sehit Rıdvan Cevik State Hospital, Van, Turkey, \\
Phone: +905419383838, e-mail: alpaslanalkan06@gmail.com \\
How to cite this article: Düzgün E, Olgun A, Karapapak M, et al. \\
Outcomes of XEN Gel Stent Implantation in the Inferonasal Quadrant \\
after Failed Trabeculectomy. J Curr Glaucoma Pract 2021;15(2):64-69. \\
Source of support: Nil \\
Conflict of interest: None
\end{tabular}

research has investigated the outcomes of inferonasal implantation after failed trabeculectomy.

The objective of our study was to evaluate the outcomes of XEN45 stent implantation in the inferonasal quadrant in patients with uncontrolled IOP after prior failed trabeculectomy.

\section{Materials and Methods}

This retrospective clinical study adhered to the tenets of the Declaration of Helsinki and was approved by the Local Ethics Committee (LEC). The requirement for informed consent was waived by the LEC. The electronic health records were reviewed and patients with refractory glaucoma who underwent XEN surgery 
after a failed trabeculectomy between September 2016 and June 2018 were included.

We included patients with open-angle glaucoma (OAG), eyes not reaching the target IOP despite trabeculectomy under maximal medication. Exclusion criteria included blepharitis, the presence of diabetes mellitus, angle-closure glaucoma, and history of uveitis or ocular trauma resulting in conjunctival scarring.

Patients underwent a complete preoperative clinical examination that included:age, gender, best-corrected visual acuity (BCVA), adjusted IOP (using Goldmann applanation tonometry), gonioscopy, anterior segment, and retinal examination including evaluation of the optic nerve head cupping. The type of glaucoma, history of surgery, and medications (a fixed combination agent was counted as two medications) were also recorded. The BCVA was expressed according to the Snellen charts. The preoperative IOP was the mean value of the last two measurements before surgery.

All patients were evaluated before the procedure and 1 day, 1 month, 3 months, 6 months, and 12 months after XEN gel implantation. Complete success criteria were defined as a postoperative IOP of $<18 \mathrm{~mm} \mathrm{Hg}$ with a $>20 \%$ reduction from preoperative value without medication or further surgical interventions (except needling). Qualified success was defined as a postoperative IOP of $<18 \mathrm{~mm} \mathrm{Hg}$ with a $>20 \%$ decrease from baseline, with or without medication, and without any secondary intervention (except needling) at the 12-month follow-up.

\section{XEN45 Gel Stent Implantation Procedure}

All operations were performed as a stand-alone procedure by the same glaucoma specialist under topical anesthesia. After standard surgical preparation, the target point was marked in the inferonasal quadrant, $3 \mathrm{~mm}$ from the limbus. The surgeon aimed to insert the stent as inferiorly as possible, as far as the speculum or eyebrow technically allowed. Ten minutes before the procedure, $0.1 \mathrm{~mL}$ of Mitomycin C solution $(0.01 \% \mathrm{MMC}, 10 \mu \mathrm{g})$ was injected into the inferonasal sub-Tenon's space, 3-4 mm away from the target point. In cases without MMC, hydrodissection was performed with a balanced salt solution (BSS). After a $19 \mathrm{~g}$ clear corneal incision from the temporal limbus, preservative-free $1 \%$ lidocaine and a cohesive viscoelastic was applied into the anterior chamber. The preloaded injector of the XEN45 gel stent was placed through the superior temporal corneal incision. The injector needle was then directed across the anterior chamber where the implantation was planned in the lower quadrant. After the needle was inserted through the iridocorneal angle, it was pushed forward in an intrascleral pathway. When the needle tip was seen under the conjunctiva, it was rotated to the side and then the stent was released. The injector was removed, and the location of the stent was controlled. After extraction of the viscoelastic material, irrigation was performed to ensure bleb formation. The surgery was ended by hydrating corneal incision.

All patients used topical moxifloxacin (0.5\%) and prednisolone acetate (1\%) eye drops, 4 times a day. Topical steroid dosage was tapered 4 weeks after surgery. Anti-glaucoma medication was completely stopped after surgery. During the follow-up period, needling or trabeculectomy decisions were given at the discretion of the same specialist. The needling was performed under topical anesthesia without any additional drug. Subconjunctival adhesions were released by moving a 27 -gauge needle. Ocular examination data, intraoperative and postoperative complications were obtained through chart review.

\section{Statistical Analysis}

All statistical analyzes were performed using Statistical Package for the Social Sciences (SPSS, Inc., Chicago, IL, USA), version 25. The descriptive statistics applied were count and percentage for categorical variables, and mean, the standard deviation for numeric variables. Rates within the groups were compared using the paired $t$-test. As the numerical variables did not follow a normal distribution, independent two-group comparisons were made using the Wilcoxon signed-rank test. A $p$ value $<0.05$ was taken to be statistically significant.

\section{Results}

The XEN gel stent was implanted into 14 glaucoma patients (9 females, 5 males) with insufficient IOP control despite trabeculectomy. The mean age of the patients was $63.4 \pm 9.7$ (52-80) years. Eight patients had pseudoexfoliative glaucoma and six were primary OAG cases. One case had undergone more than one surgery [two trabeculectomy and an Ex-Press shunt (Alcon Laboratories, Ft. Worth, TX, USA)] surgery. All patients underwent a stand-alone XEN stent implantation procedure. Nine patients were treated with MMC-augmented implantation. Mitomycin C could not be used in five patients due to the shortage of the drug supply. All patients completed all follow-up visits (from day 1 to month 12). The mean follow-up duration was 14.2 (range 12-18) months. Demographics and baseline data of the patients are shown in Table 1.

Table 2 shows the IOP levels and the change in the number of medications over time. There was a statistically significant reduction in the IOP values and medication use compared with preoperative values at all time intervals. The mean IOP was significantly reduced from a mean baseline value of $24.14 \pm 2.74 \mathrm{~mm} \mathrm{Hg}$ to a mean value of $9.43 \pm 3.41 \mathrm{~mm} \mathrm{Hg}$ on day 1, $12.64 \pm 4.63 \mathrm{~mm} \mathrm{Hg}$ at month 1 , $12.00 \pm 3.74 \mathrm{~mm} \mathrm{Hg}$ at month 3 , and $13.85 \pm 3.24 \mathrm{~mm} \mathrm{Hg}$ at month 6. The mean postoperative IOP was $12.23 \pm 2.89 \mathrm{~mm} \mathrm{Hg}$ after 12 months of follow-up, which represents a $49.3 \%$ decrease (Fig. 1).

The mean number of baseline medications was $3.71 \pm 0.47$, reducing to a mean number of $1.31 \pm 1.55$ medication used after 12 months of follow-up, which represents a $61 \%$ decrease. Seven (50\%) patients were medication-free for at least 12 months. Complete success was achieved in $50 \%$ of patients, while $85.7 \%$ showed qualified success.

The most frequent complication was transient slight intracameral hemorrhage (five eyes, 35.7\%) during surgery. Three patients (21.4\%) had numerical hypotony (IOP $<5 \mathrm{~mm} \mathrm{Hg}$ without complications) which resolved spontaneously within the early postoperative period, with no long-term consequence. Postoperatively, six eyes (42.8\%) required a single bleb needling to achieve further IOP reduction in the eyes with failed bleb. Five patients underwent a needling procedure during the 1st month and one patient during the 3rd month. Of these, two eyes (14.3\%) were managed with superior re-trabeculectomy during the 4th month and the 12th month due to failure to achieve the targeted IOP despite needling. There were no cases of vision loss, stent exposure, hypotony, choroidal effusion, lower eyelid malposition, bleb dysesthesia, or bleb-related infection (Fig. 2).

\section{Discussion}

This study is the first case series in which XEN gel implantation was undertaken in the inferonasal quadrant after failed trabeculectomy. 
Table 1: Demographics and clinical characteristics of patients

\begin{tabular}{lll}
\hline Gender, $n$ (\%) & Male & $5(35.7)$ \\
& Female & $9(64.3)$ \\
Age, mean \pm SD (range) & & $63.4 \pm 9.7(52-80)$ \\
Operated eye, $n$ (\%) & Right & $5(35.7)$ \\
& Left & $9(64.3)$ \\
Preoperative lens status, $n$ (\%) & Phakic & $9(64.3)$ \\
& Pseudophakic & $5(35.7)$ \\
Operated eye, $n$ (\%) & Aphakic & $0(0)$ \\
& Right & $5(35.7)$ \\
Diagnosis, $n$ (\%) & Left & $9(64.3)$ \\
Preoperative BCVA, mean \pm SD (LogMAR) & Primary open-angle glaucoma & $6(42.9)$ \\
Preoperative IOP, mean \pm SD (mm Hg) & Pseudoexfoliative glaucoma & $8(57.1)$ \\
Number of glaucoma medications, mean \pm SD (range) & & $0.16 \pm 0.21$ \\
Cup/disk ratio, mean \pm SD (range) & & $24.14 \pm 2.7$ \\
Antimetabolites, $n$ (\%) & & $3.71 \pm 0.4(3-4)$ \\
History of previous surgery, $n$ (\%) & & $0.71 \pm 0.19(0.5-0.9)$ \\
& MMC used & $9(64.3)$ \\
\hline BCVA, best-correct & MMC not used & $5(35.7)$ \\
\hline
\end{tabular}

BCVA, best-corrected visual acuity; IOP, intraocular pressure; Log MAR, logarithm of the minimum angle of resolution; SD, standard deviation; $M M C$, mitomycin $C$

${ }^{\dagger} \mathrm{A}$ patient had had both trabeculectomy and Ex-Press shunt surgery

Table 2: Mean intraocular pressure and mean number of glaucoma medications through 12 months postoperatively

\begin{tabular}{|c|c|c|c|c|c|c|}
\hline \multirow[b]{2}{*}{ Time } & \multicolumn{3}{|c|}{ Intraocular pressure $(\mathrm{mm} \mathrm{Hg})$} & \multicolumn{3}{|c|}{ Number of glaucoma medications ( $n$ ) } \\
\hline & Mean $\pm S D$ & Reduction (\%) & $p$ & Mean $\pm S D$ & Reduction (\%) & $p$ \\
\hline Preoperative & $24.14 \pm 2.74$ & & & $3.71 \pm 0.47$ & & \\
\hline 1 day & $9.43 \pm 3.41$ & 67.5 & $<0.001$ & 0 & 96.6 & 0.001 \\
\hline 1 month & $12.64 \pm 4.63$ & 58.2 & $<0.001$ & $1.14 \pm 1.61$ & 71.6 & 0.002 \\
\hline 3 months & $12.00 \pm 3.74$ & 58.1 & $<0.001$ & $0.79 \pm 1.31$ & 69.8 & 0.001 \\
\hline 6 months & $13.85 \pm 3.24$ & 54.1 & $<0.001$ & $1.08 \pm 1.50$ & 63.7 & 0.003 \\
\hline 12 months & $12.23 \pm 2.89$ & 49.3 & $<0.001^{*}$ & $1.31 \pm 1.55$ & 61.0 & 0.003 \\
\hline
\end{tabular}

SD, standard deviation; $\mathrm{P}$, Wilcoxon test; *Paired $t$-test

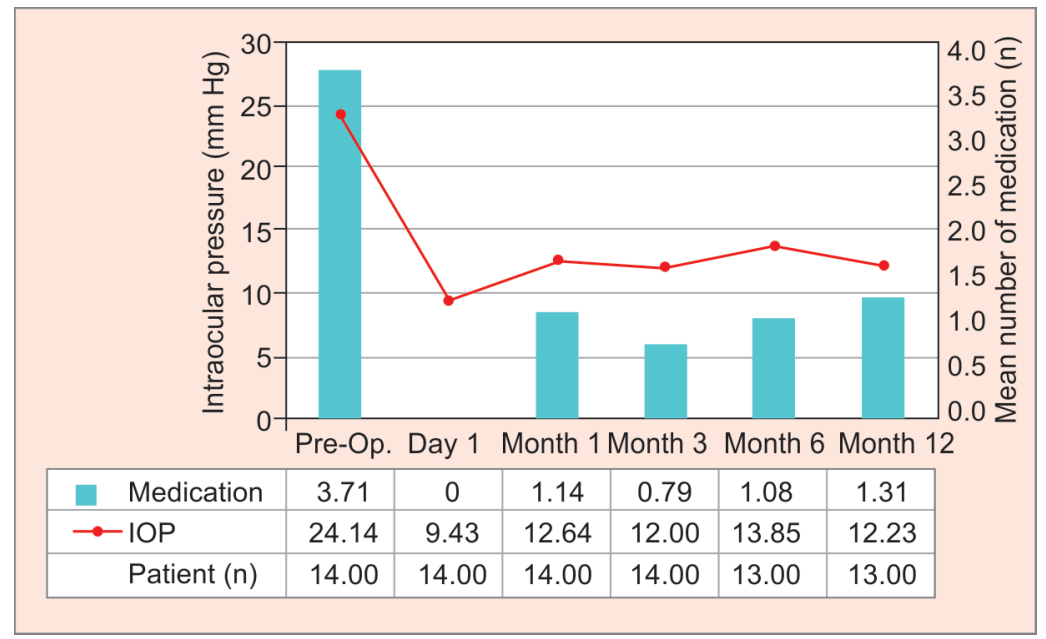

Fig. 1: Graph shows the change in mean IOP and number of medications from baseline to the 12-month follow-up visit. IOP, intraocular pressure 

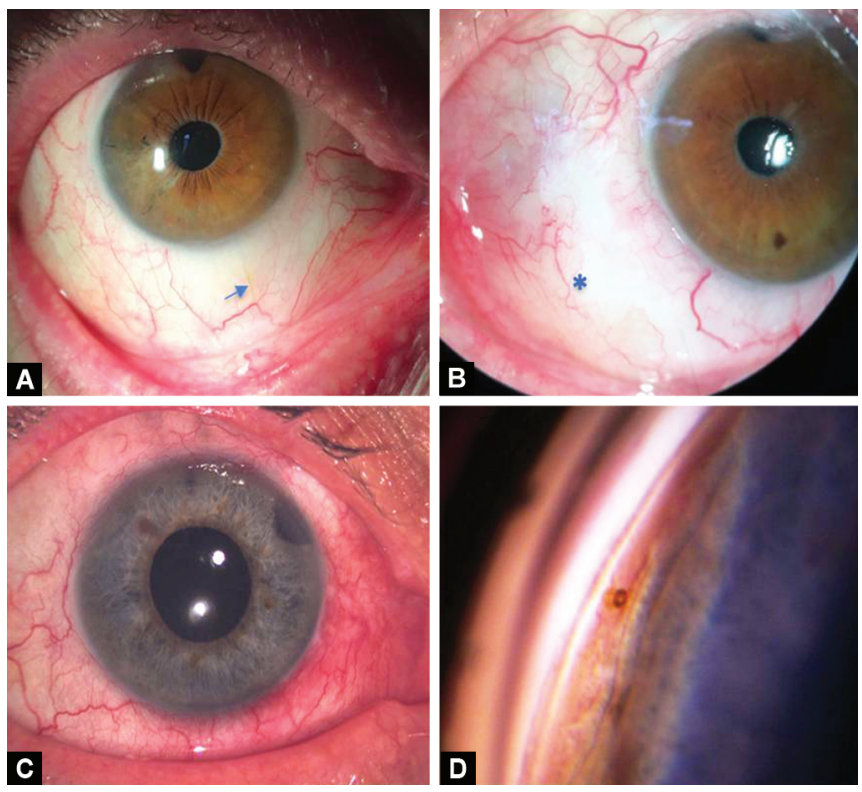

Figs $2 A$ to $D$ : (A) The XEN gel stent (arrow) is visible under the conjunctiva with a flat bleb in the right eye; (B) The appearance of an inferonasally located diffuse filtering bleb $\left(^{*}\right)$ in the left eye at month 12; (C) Anterior segment photography after XEN stent surgery in the patient who had undergone two failed trabeculectomies and Ex-Press shunt surgery; (D) View of the XEN gel stent in the iridocorneal angle on gonioscopy in the same patient

The first-year results showed that the inferonasal implantation of the XEN stent was effective in terms of IOP and anti-glaucoma medication reduction. We also observed that half of the cases achieved target IOP without eye medication at 12 months. We achieved a $49.3 \%$ mean IOP reduction and $61 \%$ glaucoma medication reduction at month 12 from baseline. The changes in IOP and medications were also comparable to outcomes of previous studies with XEN stent. ${ }^{4,5,11}$

Traditionally, GDD implantation is the preferred surgical approach in patients with failed trabeculectomy. ${ }^{12}$ Subsequent procedures may also include a bleb needling, revision, or a second trabeculectomy. However, all surgical approaches have potential challenges that are often specific to the procedure and are generally have a higher probability of failure than primary trabeculectomy. ${ }^{13}$ The patients in the current study were suitable candidates for a repeat trabeculectomy or a tube shunt surgery as a second surgical option. In the light of studies examining the effect of XEN stent on the corneal endothelium, it may be a good alternative to traditional surgeries, especially considering endothelial cell loss caused by repetitive surgeries. ${ }^{14}$ Our clinical experience and available literature have suggested that XEN gel stent surgery is less invasive, easier, and faster to apply, with a low rate of severe complications, hence we have been encouraged to prefer it in these patients.

The superonasal region is the suggested quadrant for XEN implantation. ${ }^{4,5,15}$ However, a new bleb that is created adjacent to a superiorly located, failed bleb after a trabeculectomy can affect the bleb function and increase the risk of complications due to changes in tenon and conjunctival tissue. Functional bleb formation may not occur due to fibrosis and adhesions, which may indicate the need for needling, second revision surgery, or increase the probability of failure. The application of a second antimetabolite for XEN gel stent or GDDs to the same site may further increase conjunctival damage.
It is necessary to pay attention to the potency, concentration, and exposure time of the selected antimetabolite due to differences in the wound healing response in sequential surgeries. Consequently, the conjunctival support of the implant will be weakened, and the extrusion of the stent will be unavoidable. The earlier reports described implant exposure in patients who underwent XEN implantation in the nasal quadrant close to a scarred bleb after a prior failed glaucoma procedure. ${ }^{16,17}$ XEN stent exposure poses a great risk for late-onset leakage, blebitis, or endophthalmitis. In addition, placing the stent more nasally, further away from the scar tissue may provide surgical convenience. However, a hypertrophic intra-palpebral bleb formation or dysesthesia may occur in nasal localized blebs after XEN surgery or as previously documented after trabeculectomy. ${ }^{18-20}$ It is emphasized that the XEN stent should be implanted as superiorly as possible to minimize the mentioned risks. ${ }^{18}$ In patients with healthy and mobile upper nasal conjunctiva, we preferred the upper side as generally recommended. However, all of the cases in our study were glaucoma patients who had undergone trabeculectomy and had a conjunctival scar in the upper nasal quadrant or adjacent. Therefore, we targeted the inferonasal quadrant as the most appropriate site for the implantation of the stent.

Previous studies reported that the placement of GDD inferiorly has been demonstrated to be safe and effective if there was a conjunctival scarring or a preexisting GDD in the superior quadrant. ${ }^{21,22}$ There are very few articles regarding the efficacy of the XEN stent in patients with failed glaucoma filtration surgeries for refractory glaucoma. Karimi et al. ${ }^{7}$ reported that the XEN stent might be an option in this group of glaucoma patients when the superonasal conjunctiva is spared. Previous studies reported similar rates in reduction effects for IOP, the number of medications, bleb management, and complications in cases with or without different types of failed glaucoma interventions. ${ }^{10,23}$ Sandhu and Dorey ${ }^{8}$ reported that a patient with juvenile OAG who underwent implantation of Ahmed glaucoma valves twice, after a failed trabeculectomy, has achieved adequate IOP reduction after XEN surgery. It has been reported that a patient with refractory neovascular glaucoma was successfully managed with a XEN stent after cyclophotocoagulation failed and other surgical options were unsuitable. ${ }^{9}$

Although it is difficult to report a detailed safety analysis due to the low number of patients, inferonasal implantation is well tolerated and no new serious safety concerns have been identified differently from the classical localization. There was no vision loss or sight-threatening complication. Inferonasal implantation slightly complicates the surgical procedure in some cases due to the eyebrow. We think the success rate in our small case series is acceptable, especially given the likelihood that these patients have refractory glaucoma and require more invasive surgeries. Another advantage of the stent that should be kept in mind is that it does not pose a challenge to possible subsequent filtration surgeries. In our series, secondary surgeries were required in two cases due to uncontrolled IOP at 4 or 12 months in each case. We suggest that inferonasal XEN stent implantation is an effective option for eyes with failed trabeculectomy, averting conventional incisional surgery in $85.7 \%$ of glaucoma cases.

The XEN gel stent shows promise for patients with glaucoma, but the procedure has been associated with several complications. ${ }^{4-6,15,23-25}$ Although these complications are rare, some of them may result in severe vision loss. Unfortunately, bleb- 
related infections after XEN surgery have been reported in the upper quadrant blebs in a few cases. ${ }^{25}$ In our study, we were concerned about the inferiorly located formation of the subconjunctival bleb and the use of $\mathrm{MMC}$ as a risk factor. The presumed risk factors for bleb-related infections have been evaluated in several studies. ${ }^{26-28}$ However, all reports indicate that the risk of endophthalmitis in the inferior filtering blebs is associated with trabeculectomy. ${ }^{27,28}$ Caronia et al. ${ }^{28}$ recommended trabeculectomy at the inferior limbus as a possible option for eyes in which the surgical options are limited. However, the filtration blebs associated with the XEN gel stent show some differences from those associated with trabeculectomy. ${ }^{29}$ In contrast to the thin-walled perilimbal bleb of a trabeculectomy, the XEN stent usually creates a low, posterior and diffuse bleb formation. ${ }^{18}$ Consequently, bleb morphology is not only an important indicator for IOP control after glaucoma surgery but also a predictor of bleb-related complications. ${ }^{29,30}$

Although XEN surgery does not require conjunctival or scleral dissection or sutures, the anti-scarring medications that we use with trabeculectomy are often applied to achieve functional blebs. In our study, we applied MMC as the most frequently used agent through subconjunctival injection. However, despite the application of MMC, subconjunctival fibrosis may form around the stent. As mentioned in prior studies, needling was not considered a second surgical intervention. We do not prefer to use a second MMC injection while needling to avoid possible complications. The bleb needling rate (42.8\%) of our study was comparable to those previously published reports. ${ }^{4,15}$ In the comparative study by Hengerer et al.," ${ }^{11}$ similar success rates were reported between inferonasal and superonasal implantation. However, the needling rate was lower in the inferior quadrant (27\% against $45 \%)$. They attributed this positive effect to the lower tension of the lower eyelid. As a result, to minimize complications, patients should be evaluated for the presence of uncontrolled diabetes, eyelid deformities, and blepharitis. Similarly, preoperative conjunctival assessment in the implantation site is required to achieve adequate IOP reduction and for the success of XEN surgery.

Several limitations of this study should be noted. First of all, the population of the retrospective study included only a small group of glaucoma patients with a limited duration of follow-up. In some cases, MMC could not be used during surgery or needling due to the shortage of the drug supply. These differences between cases may affect the interpretation of the results. A detailed comparative analysis was not evaluated due to low numbers in the groups with and without MMC.

\section{ConcLusion}

The current study reveals that the XEN stent implantation in the inferior quadrant can be considered a viable surgical option for glaucoma patients with a history of failed trabeculectomy who require further IOP lowering. Additional prospective studies with larger patient cohorts are required to assess long-term success and to better characterize the late-onset complications after XEN surgery.

\section{Clinical Significance}

This is the first case series describing the outcome of inferonasal implantation of XEN stent following failed trabeculectomy.

\section{References}

1. Addis $V$, Zhang M, Miller-Ellis E. New glaucoma surgical procedures. Adv Ophthalmol Optom 2016;1(1):389-410. DOI: 10.1016/j. yaoo.2016.03.009.

2. Lewis RA. Ab interno approach to the subconjunctival space using a collagen glaucoma stent. J Cataract Refract Surg 2014;40(8):13011306. DOI: 10.1016/j.jcrs.2014.01.032.

3. Tan S, Walkden A, Au L. One-year result of XEN45 implant for glaucoma: efficacy, safety, and postoperative management. Eye 2018;32(2):324-332. DOI: 10.1038/eye.2017.162.

4. Schlenker MB, Gulamhusein $\mathrm{H}$, Conrad-Hengerer I, etal. Efficacy, safety, and risk factors for failure of standalone ab interno gelatin microstent implantation versus standalone trabeculectomy. Ophthalmology 2017;124(11):1579-1588. DOI: 10.1016/j.ophtha.2017.05.004.

5. Hengerer FH, Kohnen T, Mueller M, et al. Ab interno gel implant for the treatment of glaucoma patients with or without prior glaucoma surgery: 1-year results. J Glaucoma 2017;26(12):1130-1136. DOI: 10.1097/IJG.0000000000000803.

6. Grover DS, Flynn WJ, Bashford KP, et al. Performance and safety of a new ab interno gelatin stent in refractory glaucoma at 12 months. Am J Ophthalmol 2017;183:25-36. DOI: 10.1016/j.ajo.2017.07.023.

7. Karimi A, Hopes M, Martin KR, et al. Efficacy and safety of the ab-interno Xen gel stent after failed trabeculectomy. J Glaucoma 2018;27(10):864-868. DOI: 10.1097/IJG.0000000000001044.

8. Sandhu S, Dorey MW. Case report: Xen ab interno gel stent use in a refractory glaucoma patient with previous filtration surgeries. J Glaucoma 2018;27(3):e59-e60. DOI: 10.1097/IJG.0000000000000864.

9. Tailor R, Lalias T. A case of refractory neovascular glaucoma treated with a XEN 45 implant. J Glaucoma 2018;27(10):929-930. DOI: 10.1097/ IJG.0000000000001033.

10. Hengerer FH, Auffarth G, Conrad-Hengerer I. Comparison of minimally invasive XEN45 gel stent implantation in glaucoma patients without and with prior interventional therapies. Ophthalmol Ther 2019;8(3):447-459. DOI: 10.1007/s40123-019-0193-7.

11. Hengerer FH, Auffarth G, Conrad-Hengerer I. Nasal superior versus nasal inferior implantation of the Xen 45 gel stent - one year results. J Ophthalmol Vision Res 2019;1(1):1-7.

12. Saheb H, Gedde SJ, Schiffman JC, et al. Outcomes of glaucoma reoperations in the tube versus trabeculectomy (TVT) study. Am J Ophthalmol 2014;157(6):1179-1189. DOI: 10.1016/j.ajo.2014.02.027.

13. Gedde SJ, Herndon LW, Brandt JD, et al. Postoperative complications in the tube versus trabeculectomy (TVT) study during five years of follow-up. Am J Ophthalmol 2012;153(5):804-814.e1. DOI: 10.1016/j. ajo.2011.10.024.

14. Olgun A, Duzgun E, Yildiz AM, et al. XEN Gel Stent versus trabeculectomy: short-term effects on corneal endothelial cells. Eur J Ophthalmol 2020;31(2):346-353. DOI: 10.1177/1120672120924339.

15. Widder RA, Dietlein TS, Dinslage S, et al. The XEN45 Gel Stent as a minimally invasive procedure in glaucoma surgery: success rates, risk profile, and rates of re-surgery after 261 surgeries. Graefes Arch Clin Exp Ophthalmol 2018;256(4):765-771. DOI: 10.1007/s00417-0183899-7.

16. Fea A, Cannizzo PM, Consolandi G, et al. Managing drawbacks in unconventional successful glaucoma surgery: a case report of stent exposure. Case Rep Ophthalmol Med 2015;2015:847439. DOI: 10.1155/2015/847439.

17. Galal A, Bilgic A, Eltanamly $R$, et al. XEN glaucoma implant with mitomycin C 1-year follow-up: result and complications. J Ophthalmol 2017;2017:5457246. DOI: 10.1155/2017/5457246.

18. Sekaran A, Karimi A, Lindfield D. Hypertrophic dysaesthetic blebs following ab-interno gel stent (Xen) glaucoma surgery: management and 'redirect'revision surgery. Clin Exp Ophthalmol 2018;46(9):10931095. DOI: 10.1111/ceo.13335.

19. Fernandez-Garcia A, Romero C, Garzon N. "Dry Lake" technique for the treatment of hypertrophic bleb following XEN ${ }^{\circledR}$ gel stent 
placement. Arch Soc Esp Oftalmol (English Edition) 2015;90(11):536538. DOI: 10.1016/j.oftal.2015.03.003.

20. Budenz DL, Hoffman K, Zacchei A. Glaucoma filtering bleb dysesthesia. Am J Ophthalmol 2001;131(5):626-630. DOI: 10.1016/ s0002-9394(00)00901-6.

21. Martino AZ, Iverson S, Feuer WJ, et al. Surgical outcomes of superior versus inferior glaucoma drainage device implantation. J Glaucoma 2015;24(1):32. DOI: 10.1097/IJG.0b013e318287ac8f.

22. Rachmiel R, Trope GE, Buys YM, et al. Intermediate-term outcome and success of superior versus inferior Ahmed glaucoma valve implantation. J Glaucoma 2008;17(7):584-590. DOI: 10.1097/ IJG.0b013e31816299bc.

23. Karimi $A$, Lindfield $D$, Turnbull $A$, et al. A multi-centre interventional case series of 259 ab-interno Xen gel implants for glaucoma, with and without combined cataract surgery. Eye 2019;33(3):469-477. DOI: 10.1038/s41433-018-0243-8.

24. Mansouri K, Guidotti J, Rao HL, et al. Prospective evaluation of standalone XEN gel implant and combined phacoemulsification-XEN gel implant surgery: 1-year results. J Glaucoma 2018;27(2):140-147. DOI: 10.1097/IJG.0000000000000858.
25. Olgun $A$, Imamoglu $S$, Karapapak $M$, et al. Endophthalmitis after XEN gel stent implantation: 2 cases. J Glaucoma 2018;27(12):e191-e194. DOI: 10.1097/JJG.0000000000001076.

26. Kim E-A, Law SK, Coleman AL, et al. Long-term bleb-related infections after trabeculectomy: incidence, risk factors, and influence of bleb revision. Am J Ophthalmol 2015;159(6):1082-1091. DOI: 10.1016/j. ajo.2015.03.001.

27. Soltau JB, Rothman RF, Budenz DL, et al. Risk factors for glaucoma filtering bleb infections. Arch Ophthalmol 2000;118(3):338-342. DOI: 10.1001/archopht.118.3.338.

28. Caronia RM, Liebmann JM, Friedman R, et al. Trabeculectomy at the inferior limbus. Arch Ophthalmol 1996;114(4):387-391. DOI: 10.1001/ archopht.1996.01100130383004.

29. Lenzhofer M, Strohmaier C, Hohensinn M, et al. Longitudinal bleb morphology in anterior segment OCT after minimally invasive transscleral ab interno glaucoma gel microstent implantation. Acta Ophthalmol (Copenh) 2019;97(2):e231-e237. DOI: 10.1111/aos.13902.

30. Picht G, Grehn F. Classification of filtering blebs in trabeculectomy: biomicroscopy and functionality. Curr Opin Ophthalmol 1998;9(2):28. DOI: 10.1097/00055735-199804000-00002. 